\title{
High-resolution neutron three axis diffractometer for studies of elastic and plastic deformation of polycrystalline materials
}

\author{
P. Mikula, V. Ryukhtin, J. Šaroun and P. Strunz \\ Nuclear Physics Institute CAS, 25068 Řež, Czech Republic \\ mikula@ujf.cas.cz,ryukhtin@ujf.cas.cz,saroun@ujf.cas.cz,strunz@ujf.cas.cz
}

In our contribution we will present feasibility of using a high-resolution three axis neutron diffractometer performance for elastic and plastic deformation studies of metallic polycrystalline samples. The method consists of unconventional set-up using bent perfect crystal (BPC) monochromator and analyzer with a polycrystalline sample in between (see Fig. 1). After realization of focusing conditions in real and momentum space [1] at the neutron wavelength of $0.162 \mathrm{~nm}$, a high-resolution down to $F W H M(\Delta d / d)=2 \times 10^{-3}$ and $F W H M(\Delta d / d)=3 \times 10^{-3}$ has been achieved on $2 \mathrm{~mm}$ diameter standard $\alpha-\mathrm{Fe}(110)$ sample in the vertical position and $8 \mathrm{~mm}$ diameter one for $10 \mathrm{~mm}$ irradiated length in the horizontal position, respectively. This experimental set-up opens the possibility for measurements of small lattice parameter changes even on bulk samples $[2,3]$. The drawback of the performance shown in Fig. 1 consists in the necessity of the step-by-step analysis by rocking the BPC-analyzer. However, in the case of residual strain/stress
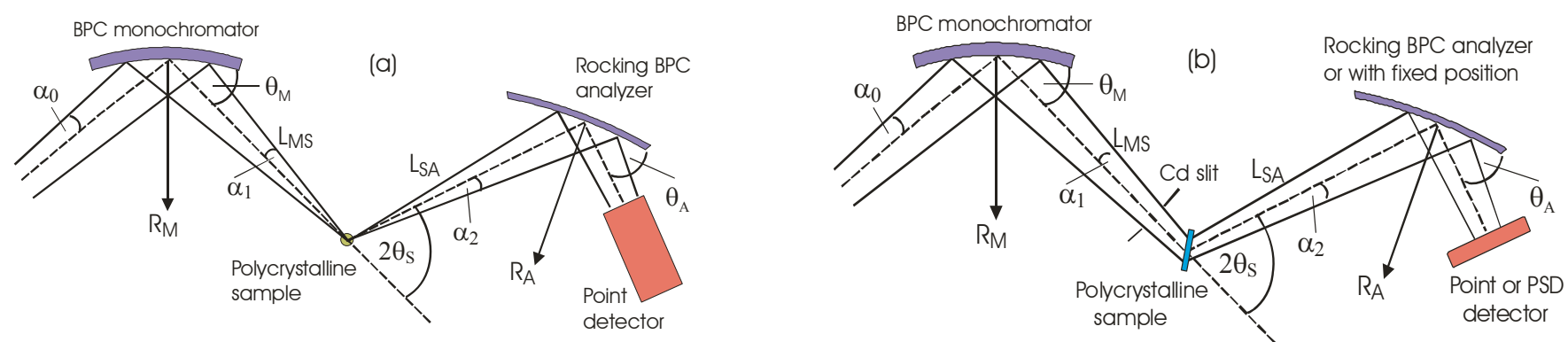

Figure 1. Three axis diffractometer set-ups employing BPC monochromator and analyzer as used in the experimental feasibility studies ( $R_{\mathrm{M}}, R_{\mathrm{A}}$ - radii of curvature, $\theta_{\mathrm{M}}, \theta_{\mathrm{A}}$ - Bragg angles) with a point detector - (a) and/or with a position sensitive detector (PSD) - (b).

measurements, position sensitive detector with the fixed position of the analyzer can by employed (see Fig. 1b) which partly eliminates this drawback. The presented sut-up permits the investigation of structure changes of bulk samples of the volume of several cubic centimeters, e.g. in the tension/compression rig, with a high resolution. Thus it provides average values of microstructure characteristics over the irradiated gauge volume, e.g., as a function of macroscopic strain loaded on the sample by an auxiliary instrument (including the oscillating loads). It also permits microstrain studies (root-mean-square microstrains) as well as the effective grain size as a function of macroscopic strain from applying shape analysis of neutron diffraction line [4].The feasibility of the instrument set-up will be demonstrated on the polycrystalline samples of standard and low carbon shear deformed steel wires. It can be stated that the presented three-axis method can offer further complementary information to that achieved by the other methods commonly used.

[1] Vrána, M., Lukáš, P., Mikula, P. \& Kulda, J. (1994). Nucl. Instrum. Methods in Phys. Research, A 338, 125.

[2] Mikula, P., Šaroun, J., Ryukhtin, V. \& Stammers, J. (2020). Powder diffraction, 35, Issue 3, 185.

[3] Mikula, P., Šaroun, J., Ryukhtin, V. \& Strunz, P. (2020). Materials - MDPI, 13, 5449.

[4] Davydov, V., Lukáš, P., Strunz, P. \& Kužel, R. (2008). Mat. Sc. Forum, 571-572, 181.

\section{Keywords: Neutron diffraction; high resolution; strain/stress measurement; three axis set-up}

Measurements were carried out at the CANAM infrastructure of the NPI CAS Řež. P. Mikula and J. Šaroun acknowledge support from ESS participation of the Czech Republic - OP (CZ.02.1.01/0.0/0.0/16_013/0001794) and from the project ESS Scandinavia-CZ II (LM2018111), respectively. Furthermore P. Strunz and V. Ryukhtin acknowledge support from the Czech Academy of Sciences in the frame of the program "Strategie AV21, No. 23". The authors thank B. Michalcová from NPI CAS for significant help with measurements and data elaborations. 